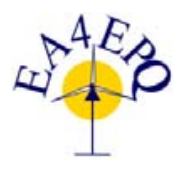

International Conference on Renewable Energies and Power Quality (ICREPQ'16)

Madrid (Spain), $4^{\text {th }}$ to $6^{\text {th }}$ May, 2016

Renewable EEnergy and Power Quality. Tournal (RE\&PQJ)

ISSN 2172-038 X, No.14 May 2016

\title{
New Application Rules for Fuses in Low and Medium Voltage Grids having Distributed Generation
}

\author{
Juan Carlos Gómez \\ Electrical Power System Protection Institute \\ Rio Cuarto National University \\ Rio Cuarto, Argentina. \\ jcgomez@ing.unrc.edu.ar \\ (Correspondent author)
}

\author{
Daniel Humberto Tourn \\ Electrical Power System Protection Institute \\ Rio Cuarto National University \\ Rio Cuarto, Argentina.
}

\begin{abstract}
The fuse is a well-established protective device which application in distribution systems (DG) need to be re-analyzed. Distribution systems having embedded DG introduce changes into the fault current and thus in the selective coordination studies. The emerging problems are presented, new methodologies and solutions are introduced. If none of the two methodologies possesses the required ability, then the application of more sophisticated protection devices should be analyzed. With this procedure the best solution from the technical and economic points of view could be obtained.
\end{abstract}

Index Terms-Fuses, Distributed generation, Smart fuses, Selective coordination, Specific Energy.

\section{INTRODUCTION}

The fuse is the oldest protective device, near to 140 years old, and that of more diffusion now, basing its wide diffusion fundamentally on its high reliability. From their birth and until some few years ago their application was fundamentally in radial circuits [1].

The low voltage high breaking capacity fuses have been used in meshed systems like that of Figure 1, for many years, with excellent result, using for its selective coordination the methodology of correction for the time-current characteristic curves as function of the current share among devices connected to the same point (node). The fuses near to each node are of the same rated current, as well as they were similar conductor cross-sections [2].
Already in that application the load and fault currents could change their circulating direction, but their magnitude stays in a similar way in the diverse devices.

The currents possess similar wave-forms, differing only in the amplitude. The presence of a fault resistance, independently of their value, affects equally to the currents distribution, in other words it modify the magnitudes but not the distribution percentages, share that is exclusively function of the localization of the point in fault. Except for the soft changes of load in the nodes (that could affect the time of operation due to the pre-load heating of the fuse element), significant changes do not exist in the currents that circulate throughout the fuses.

As an example, Figure 1 shows that the fuse located on the upper part of the failed branch has two contributions; on the other hand the one located below has three component currents. If the fault is located very near the inferior node, the current I4 can be significantly higher than I3. The condition for selective operation between the fuses of the failed branch and those of the non-failed, it is that the end of the prearc and arc processes are reached (complete fuse operation) of the fuse of the faulted branch, without arriving to the end of the prearc (irreversibility point) of the one that should not operate (located on the non-failed branch). If one operates before, for example the I 2 before the I 3 are presented the failure of the coordination.

It should be considered especially what type of fuse is used, since in low voltage and in medium voltage are used high breaking capacity fuses that are able to control the "specific energy" value $\left(\mathrm{I}^{2} \mathrm{t}\right)$ for what it is allowed a more precise study of the coordination. In medium voltage it can be 
used the expulsion fuse whose procedure of coordination study is different and in spite of being quite well-known it does not possess the precision of the one based on $\mathrm{I}^{2} \mathrm{t}$.

The correction method is based on the traditional characteristic time-current curves (TCC) doing a movement parallel to itself based on the currents distribution or by reading the time intervals corresponding to the shared current values. Figure 2 shows the situation of the original curve of a hypothetical fuse for instance class gL rated $100 \mathrm{~A}$, when the fault current reach 10 times the rated current and considering that the fault is distributed 40 and $60 \%$ in the two fuses supplying currents to the fault, I3 and I4 respectively in the example of Figure 1.

In figure 2 it can be seen that the fuse with $100 \%$ of the fault current should act at a time of 0.07 seconds. If it is considered that the contributed currents are the same in the superior node, that is to say $50 \%$, the fuses circulated by I1 and I2 would act in $1 \mathrm{~s}$. In the inferior node with 33\% in each branch, the fuses circulated by I5, I6 and I7 would operate in $8 \mathrm{~s}$. Situation that corresponds to fault located directly on the nodes.

Under the presented situation, the fuse having current I4 circulating $600 \mathrm{~A}$ will operate in $0.5 \mathrm{~s}$, whilst the other fuses of the node, supplying 200 A would operate in $370 \mathrm{~s}$, having widely assured the selectivity. Simultaneously, fuse having current I3, initially 400 A would operate in $2 \mathrm{~s}$, and fuses of currents I1 and I2 having 200 A would open in 370 s. When current I4 operate the corresponding fuse in $0.5 \mathrm{~s}$ the whole fault current is transferred, thus the current I3 jumps from 400 A to $1000 \mathrm{~A}$, causing the fuse operation in approximately 0.6 $\mathrm{s}$ before the operation of fuses having currents I1 and I2 which would operate in approximately $1.3 \mathrm{~s}$. The selectivity is also reached.

The reverse situation, has only a difference that fuses supplying to the nodes are 3 instead of 2 and the reverse. Fuse having I3 operate in $0.5 \mathrm{~s}$ and the other fuse similarly jumps from $400 \mathrm{~A}$ to $1000 \mathrm{~A}$, operating in $0.6 \mathrm{~s}$ whilst the 3 supplying fuse would operate in $8 \mathrm{~s}$. Again the selectivity is reached.

If the current distribution is even more unbalanced, for example $80 \%$ and $20 \%$, the temporary difference (800A; $0.15 \mathrm{~s}$ ) is of only $0.08 \mathrm{~s}$ (the time for $100 \%$ of the fault current compared with the one for $80 \%$ ), which is still acceptable. But with higher unbalance, the time difference can be of the same order that the tolerances characteristic of fuses production and the result can be the coordination fail.

The fuses manufacturers, recommend (for I1 $>\mathrm{I} 2$ ) that the relationship I1 / (I1+I2) or I1/I3 do not surpass at 0.8 . If I2 is very low practically I1 and I3 are the same, being impossible the coordination. The control of the non surpassing of the 0.8 value is verified based on the section of the used conductors and to the longitude of the branches between nodes.

Besides, it should not be forgotten that the main function of the fuse is to protect some equipment or part of the circuit, and the second are to operate coordinately.

\section{OPERATION OF FUSES IN CIRCUITS HAVING DISTRIBUTED GENERATION}

Distributed generation (DG) is presently used to increase reliability and reduce (or defer) system investments. Besides, DG systems are increasingly acquiring more relevance than conventional methods due to energy requirements and the use of renewable energy sources [3, 4].

The introduction of the distributed generation as it is shown in the simple circuit of Figure 3, where for example DGS is a synchronous generator and DGPV is a photovoltaic source, totally modifies the usual protection approaches with fusible used so far, since the current now can change the direction and magnitude in gradual or sudden way.

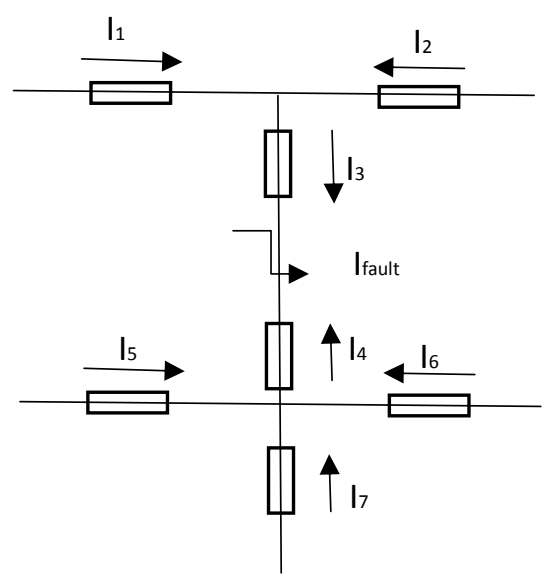

Figure 1, Typical meshed low voltage circuit

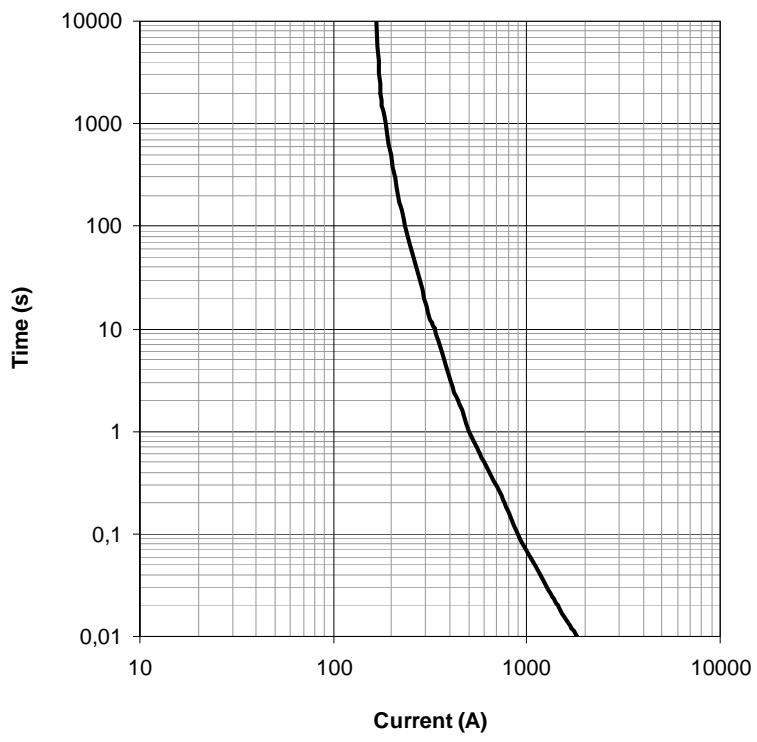

Figure 2, Traditional TCC of a gL class 100 A fuse. 


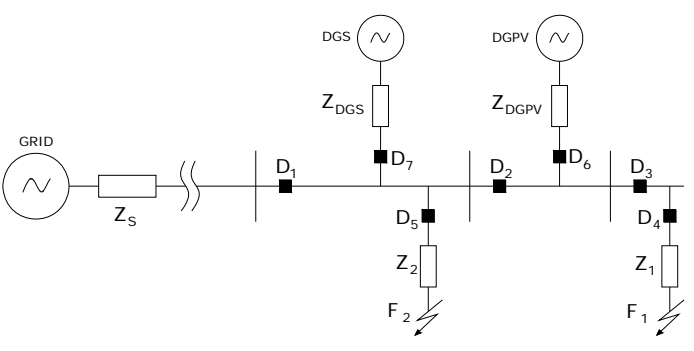

Figure 3, Distribution system simplified circuit.

The change of direction of the fault current circulating through the protective devices depends on the fault localization, besides the distributed sources can also contribute currents of magnitude variable in time. The centralized or concentrated sources contribute usually invariable fault currents. Some distributed sources possess protection that reduces the current contributed to the fault, which is normally of low magnitude (photovoltaic sources), what hinders or difficult the detection of the perturbation [5].

The classic example of this new situation is the analysis of the coordination among the protective devices D6, D2 and D5 in presence of the fault F2, shown in Figure 3. The setting of the D6 protective device, usually a circuit breaker, is fixed by regulations imposed by the utility, D2 and D5 are usually fuses whose selective coordination was not studied when installed, since it was not necessary before the connection of the source DGPV takes place.

The Figure 4 shows the typical form of the rms current of the fault F2 with the contribution of the grid, more that of the distributed synchronous generator and that of the photovoltaic cell with protection current limiter [6].

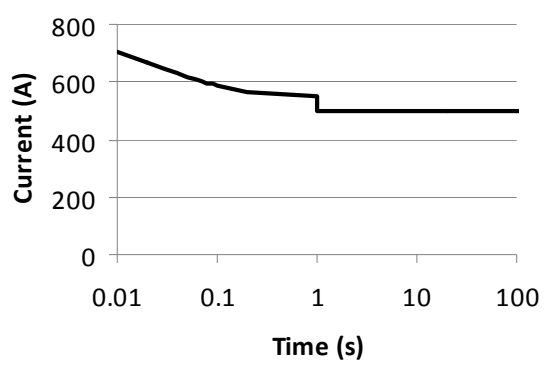

Figure 4, Short-circuit current supplied by the grid and two DG (synchronous and PV).

For the variable form of the fault current, the traditional time-current characteristic curves, are not any more applicable, thus the coordination work should be focused to the specific energy in function of the time curves $\left(\mathrm{I}^{2} \mathrm{t}-\mathrm{t}\right)$, as that of Figure 5.

The problem denominated "Blinding effect" is also presented that could be explained by studying the fault F2 in Figure 3 that is fed by the grid and for the distributed generators, therefore the fuse D2 really see a lot less than the fault current, acting according to what detects, generating coordination problems.

On the other hand, many of the distributed sources (generators or energy store devices) generate in d.c. form, using for it appropriate fuses for such a function, supplying energy to the system previous passing through the corresponding inverter. The rest of the distribution system operates in a.c., for what should be coordinated fusible devices of operative characteristic very different, what complicates and it sometimes prevents to reach a satisfactory coordination [7].

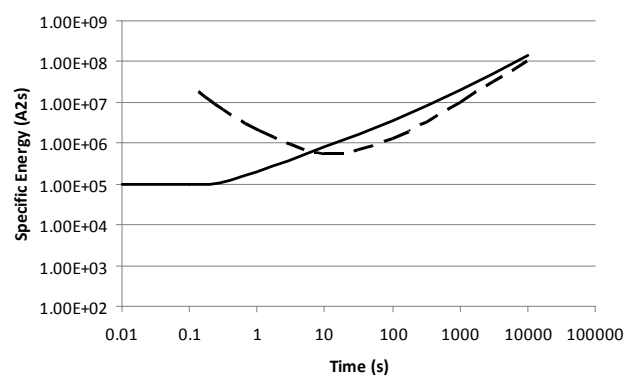

Figure 5, Specific energy as time functions for a fuse and a circuit breaker.

The protection by fuses is inherently a single-phase protection, becoming a three-phase protection by means of the employment of three-phase on-load switches with three single-phase trips, methodology widely applied in medium voltage level, but not wide spread in low voltage applications. Recently this type of three-phase low voltage device have been presented to the market.

This situation of traditional distribution systems where distributed generation has been incorporated, presents new requirements for the traditional fuses, giving place to a strong tendency to the innovation of the traditional fuses that are going to become into a device with added intelligence. It is also noticed the tendency to the substitution of the fuse by more sophisticated protection devices, what is difficult to economically justify and that also produce a significant loss of reliability.

In the specific references several definitions for intelligent or smart fuse can be found, for instance "a fuse able to act on the command of a fault detecting device and to interrupt currents below its intrinsic time-current characteristic".

The tendency to the intelligence addition began more than 80 years ago with the idea of applying chemical charges, continued by the employment of saturable transformers that today moves toward the triggered fuse (based on pyro-switch, external electric pulses, power electronics and non-lineal voltage dependent resistor).

For that explained, the following question arises: Smart fuses: are really a today need?

The distributed sources are "recently arrived" to a system that was working well, by what the less possible thing (element or methodology) should be modified. The costs 
should not act against the use of the GD, since they could lose the considerable benefits that their use generates, impelling the retirement of the interest of the investors in renewable and environmentally friendly energy.

\section{CONCLUSIONS}

It is concluded that the fuses are of great importance in the distribution systems with distributed generation. It is necessary to deeply study the today protection schemes keeping in mind the contribution of the distributed generators, by means of the employment of the traditional methodologies, of very well known use to date. In case of significant (more than $20 \%$ ) injections of energy coming from the DGs, the method of specific energy in function of the time should be applied. If none of the two methodologies possesses the required ability, only in this moment the application of the Smart fuses or of more sophisticated protection device should be analyzed. With this procedure the best solution from the technical and economic points of view will be obtained.

\section{REFERENCES}

1. H. W. Baxter, Electric Fuses, Edward Arnold and Co., London, 1950.

2. A. Wright, P. G. Newbery, Electric Fuses, Second edition, Peter Peregrinus Ltd., London, 1984.

3. J. Gómez, M. Morcos, "Overcurrent coordination in systems with distributed generation," Electric Power Components and Systems, Vol. 39, No. 6, pp. 576 - 589, 2011.

4. IEEE Standard 1547, IEEE Standard for Interconnecting Distributed Resources with Electric Power Systems, 2003.

5. F. Viawan, D. Karlsson, A. Sannino, J. Daalder, "Protection Scheme for Meshed Distribution Systems with High Penetration of Distributed Generation”, Power Systems Conference, pp. 99 - 104, 2006.

6. J. C. Gomez, Fusibles eléctricos: aplicaciones prácticas y su justificación teórica, EDIGAR, Buenos Aires, Argentina, 2012.

7. R. Chabanloo, H. Abyaneh, A. Agheli, H. Rasyegar, "Overcurrent relays coordination considering transient behaviour of fault current limiter and distributed generation in distributed power network", IET Generation, Transmission \& Distribution, Vol. 5, n 9, pp. 903-911, 2011. 